\title{
El Rol de la Universidad Estatal como un Bien Público. Retos en el Caso Colombiano
}

\author{
John J. Salinas ${ }^{(1) *}$ y Johnny Tamayo(2) \\ (1) Universidad de Caldas, Fac. de Ciencias Jurídicas y Sociales, Depto. de Economía y Administración, \\ Carrera 23 No. 58-65 Sede Palogrande, Manizales-Colombia. (e-mail: jhon.salinas@ucaldas.edu.co) \\ (2) Universidad Nacional de Colombia, Fac. de Ingeniería y Arquitectura, Depto. de Ingeniería Industrial \\ Campus La Nubia, Manizales-Colombia (e-mail: jatamayoar@unal.edu.co)
}

Recibido Ago. 5, 2017; Aceptado Oct. 10, 2017; Versión final Nov. 8, 2017, Publicado Abr. 2018

\begin{abstract}
Resumen
En este trabajo se explora y analiza el rol inherente a la universidad estatal en su función de contribuir al bien público. Se presentan algunas maneras de interpretar el bien público en la educación superior. Igualmente, se hace una breve aproximación al sistema de educación superior colombiano y a ciertas dimensiones del bien público, con el propósito de identificar los desafíos más relevantes. El análisis proporciona una perspectiva crítica sobre algunas cuestiones importantes y desafiantes con respecto al propósito público de las universidades estatales. Se concluye que una de las principales funciones de la universidad estatal para aportar al bien público se encuentra en su promesa de justicia social. En el caso colombiano, se debe continuar aumentando el acceso con equidad y calidad en todas las regiones del país a fin de disminuir las brechas existentes.
\end{abstract}

Palabras clave: bien público; universidad estatal; educación superior; educación superior colombiana; justicia social

\section{The Role of the Public University as a Public Wellness. Challenges in the Colombian case}

\begin{abstract}
This paper explores and analyzes the inherent role of the public university in its function of contributing to the public wellness. Some ways of interpreting higher education as a public good are presented. Likewise, a brief approach is made to the Colombian higher education system and to certain dimensions of the public wellness to identify the most relevant challenges. The analysis provides a critical perspective on some important and challenging issues regarding the public purpose of government universities. It is concluded that one of the main functions of public universities to contribute to the public wellness is its promise of social justice. In the case of Colombia, access with equity and quality in all regions of the country in order to reduce the existing gaps must continue.
\end{abstract}

Keywords: public wellness; public university; higher education; Colombian higher education; social justice 


\section{INTRODUCCIÓN}

En una era de globalización e internacionalización, los propósitos sociales y el valor público de la educación superior han sido moldeados por el impacto de una serie de tendencias comunes que convergen en una nueva ortodoxia sobre su valor social y la manera como debe ser gestionadas las universidades (Singh, 2012). Algunas de estas tendencias y transformaciones que han experimentado, en menor o mayor grado, los sistemas de educación superior a nivel mundial durante las últimas décadas, incluyen: a) la exigencia de que el sector demuestre eficiencia, eficacia, la implementación de sistemas externos de aseguramiento de la calidad y otros marcos de rendición de cuentas diseñados para dar cabida a un mayor control de las partes interesadas; b) la disminución de las inversiones de fondos públicos para subsidiar los costos de matrículas y servicios de los estudiantes, esta tendencia suele ir acompañada de presiones para diversificar las fuentes de financiación, reduciendo así la responsabilidad primordial del Estado con la universidad pública; c) el predominio de los enfoques gerenciales y empresariales, bajo el marco de la nueva gestión pública; d) el creciente desarrollo de reformas del currículo orientadas hacia el mercado de trabajo; e) un mayor enfoque de la financiación pública y privada hacia la investigación aplicada; f) búsqueda de mayores vínculos de la academia con la industria, la preocupación por las cuestiones de los derechos de propiedad intelectual y la priorización de la investigación para el desarrollo y la comercialización de productos (Enders y Jongbloed, 2008; Singh, 2012; Santiago et al., 2008; Sobrinho, 2009).

Bajo este contexto, la noción de bien público ha tenido una mayor resonancia como marco alternativo o complementario en los debates sobre los propósitos de la educación superior (Singh, 2014). De hecho, la educación superior tradicionalmente ha sido considerada como un bien público (Sobrinho, 2009), debido a que todo individuo que se beneficia de acceder a ella, puede contribuir al bien común mediante las habilidades, destrezas y perspectivas que adquiere (Zembylas, 2017). Asimismo, en la concepción de la educación superior como bien público existen una serie de beneficios sociales proporcionados por los graduados universitarios: el pago de mayores impuestos, menor propensión al tabaquismo, reducción de la pobreza, menor probabilidad de encarcelamiento y, por tanto, menor apoyo público a esta población. Como beneficios privados no monetarios de la educación superior se han identificado: mejor salud en el futuro, mejor desarrollo cognitivo para los hijos y mayor satisfacción laboral (Dill, 2015; McMahon, 2009; Willetts, 2015). Adicionalmente, la inversión en educación superior se ha asociado positivamente con beneficios sociales como la innovación industrial y el crecimiento económico (Santiago et al., 2008), mejorando además el desempeño de las regiones donde se concentran las universidades (Marginson, 2010).

Aunque, también se reconoce que la educación superior sostiene aspectos tanto de bien público como de privado. Calhoun (2011) anota que las universidades educan para la ciudadanía, así como para los negocios; educan para el servicio público, así como para el beneficio privado; realizan investigación avanzada y el análisis de problemas sociales de alta significación, de los que no se derivan beneficios económicos. De igual modo, todas universidades colaboran con el sector productivo, venden servicios y productos de conocimiento y, en general, aprenden a desenvolverse en los términos impuestos por el "capitalismo académico" (Brunner, 2016). En particular, las universidades privadas a menudo se orientan hacia bienes públicos, tales como la preservación y el intercambio de conocimientos, la investigación, entre otros. Por su parte, las universidades públicas reciben dinero privado, no sólo del cobro de matrículas sino también de contratos de investigación, de servicios de asesoría y consultoría, distribuyen además beneficios privados a sus egresados, como la posibilidad de acceso a empleos que generan mayores salarios e ingresos durante toda la vida. A este respecto, se puede afirmar que la educación superior distribuye beneficios privados de valor desigual, incluso, en instituciones estatales casi libres de costos de matrícula y, por tanto, nominalmente "públicas" (Marginson, 2012). Además, en un contexto globalizado, el Estado y el mercado, o lo público y lo privado, no quedan planteados como mutuamente excluyentes entre sí, teniendo en cuenta la cada vez más notoria participación de alianzas público-privadas y, la asignación de fondos concursables que no discriminan la naturaleza pública o privada de la universidad, pues los criterios a valorar son la calidad, pertinencia y viabilidad de los proyectos (Morandé, 2011).

Por tanto, la distinción entre lo público y lo privado en la educación superior se ha vuelto difusa. Más aún, los rasgos privados están creciendo en incidencia e importancia en las universidades estatales, pues las presiones financieras han llevado a una búsqueda apresurada de la diversificación de ingresos, a veces incompatibles con los valores académicos tradicionales. Por ejemplo, algunas de ellas han incursionado en la oferta de programas especiales "autosostenibles financieramente", con costos de matrículas diferenciales a las que pagan estudiantes regulares en una misma universidad. Es decir, la privatización de ciertos aspectos en estas instituciones públicas o la introducción de los denominados cuasimercados en la educación superior (Teixeira et al., 2017), que provocan ambigüedad y un debilitamiento del compromiso y la responsabilidad de la universidad pública con la sociedad (Kezar, 2015). Por este motivo, algunos analistas han señalado incongruencias en la idea de universidad estatal como una institución financiada por el Estado y sin fines de lucro, debido a que, cada vez más, los recursos tienden a provenir tanto de fuentes públicas como privadas (Singh, 2014). 
En este orden de ideas, la presente reflexión documentada tiene como objetivo explorar en la literatura analítica el papel inherente y singular de la universidad estatal en su función de contribuir al bien público, así como también, el rol que debería jugar en el desarrollo de la ciencia. Se presenta además, una breve aproximación al sistema de educación superior colombiano y a ciertas dimensiones del bien público, con el propósito de situar algunos de los conceptos desarrollados en el documento. El tema cobra relevancia en la medida que el bien público constituye una perspectiva crítica que llama la atención sobre elementos del "mercado" que se han venido instaurando dentro de universidades estatales y que distorsionan las misiones centrales de estas instituciones. De esta manera, se espera contribuir a la comprensión sobre algunas cuestiones importantes y desafiantes con respecto al propósito público de las universidades estatales. El resto del documento está estructurado de la siguiente manera: la concepción de la educación superior como bien público, un vínculo entre la universidad estatal y el bien público, la universidad estatal frente al desarrollo de la ciencia como bien público, algunas dimensiones del bien público en la educación superior colombiana y conclusiones. Es importante anotar que utilizamos el concepto de la educación superior y de universidades indistintamente, reconociendo de antemano que no son lo mismo, pues en algunos casos, como en el colombiano, la educación superior comprende los niveles de educación técnico y tecnológico, los cuales no hacen parte del presente tema de estudio.

\section{LA CONCEPCIÓN DE LA EDUCACIÓN SUPERIOR COMO BIEN PÚBLICO}

Antes de abordar la noción de la educación superior como bien público, conviene definir primero lo que este significa. Así, un bien público "puro" desde el punto de vista económico alude a un bien o servicio que se caracteriza tanto por la no rivalidad en el consumo como por la imposibilidad de exclusión, además produce beneficios a un amplio conjunto de personas, independientemente de que hayan o no pagado los costos de producirlos. La no rivalidad significa que el uso por parte de un individuo/empresa no limita el uso por parte de otra. $Y$ no excluible se refiere a que el uso no se niega a nadie (por ejemplo, el aire puro, la defensa nacional). Aunque, en realidad ambas características pueden ser impugnadas, la no rivalidad porque prácticamente ningún bien puede considerarse infinito y la no exclusión porque el acceso a todos los bienes es influenciado por factores como la riqueza, la salud, el deseo, entre otros (Scott, 2015). Aún más, la teoría económica sugiere que la rivalidad es un continuo, en lugar de una categoría binaria. Es decir, donde muchos bienes y servicios están en algún punto entre "completamente rival" y "completamente no rival" (Longden y Bélanger, 2013). Por ejemplo, la asistencia a una conferencia no es rival hasta una cierta capacidad del auditorio, después de esta, cada usuario adicional disminuye el acceso a otros. En contraste, los bienes privados son excluibles y rivales, quienes los poseen pueden ejercer sus derechos de propiedad, evitando que otros los utilicen o consuman sus beneficios (Dill, 2015).

Ahora bien, en la concepción de la educación superior como bien público es importante advertir que esta no es bien público "puro", ya que muchas personas pueden verse excluidas por múltiples causas: lugares de estudio limitados, procesos de selección, barreras económicas, entre otros. En consecuencia, lo que se asocia con educación superior como bien público es la existencia de una amplia gama de beneficios -externalidades positivas- (Baum y McPherson, 2011), algunas de ellas mencionadas líneas atrás. No obstante, cabe citar la distinción propuesta por Marginson (2011) entre bienes públicos y el bien público en la educación superior, quien define bienes públicos como los productos finales del sector que pueden ser intangibles y tomar forma individual o colectiva. Esto podría incluir una gama de productos intelectuales, sociales y económicos, como una fuerza de trabajo más productiva económicamente, aumentos en la capacidad intelectual que conducen al avance científico (Williams, 2016). A su turno, el bien público, según Marginson el enfoque es más normativo y tiende a enfatizar en las actividades, beneficios o recursos accesibles a todos; asimismo, está vinculado a la educación superior en un sentido más amplio de democratización y desarrollo humano. Es decir, incluye ciudadanos mejor informados que conducen a fortalecer la democracia y a una sociedad y un conocimiento más incluyente, concebido como un fin en sí mismo (Williams, 2016).

Williams (2016) plantea que la definición del bien público en la educación superior ha pasado a lo largo de varias décadas desde la concepción de conocimiento en sí mismo, a resultados del conocimiento que pueden utilizarse para obtener un mejor desempeño económico nacional mediante el avance científico y tecnológico; y finalmente a un enfoque de inclusión y movilidad social, en forma de empleabilidad individual, aumento de ingresos y seguridad en el empleo. En efecto, la teoría de capital humano ha reforzado esta concepción, pues considera que "la demanda de educación se deriva de las decisiones óptimas de inversión de individuos racionales" (Schoenenberger, 2005). Lo cual tiene implicaciones en la relación entre las universidades y el Estado, conllevando a una ideología más individualista que sugiere que los graduados obtienen beneficios económicos de su educación universitaria, y en consecuencia, ha hecho que parezca razonable que éstos deban contribuir más a su costo (Popp y Paradisse, 2016); forzando un debate, en esencia, sobre quién obtiene los mayores beneficios -o bienes- de la educación superior, los individuos o la sociedad (Hensley et al., 2013). 
Para Marginson (2007) el análisis de beneficios económicos mediante tasas de retorno a la inversión en la educación superior tiende a minimizar o ignorar los beneficios colectivos, los cuales no se reducen a beneficios individualizados con un valor monetario. En esta misma línea, Scott (2015) sugiere que todos o la mayoría de los beneficios individuales se ven reforzados por beneficios sociales más amplios, de modo que, en lugar de considerar beneficios privados y públicos como un juego de suma cero, es posible hacer hincapié en sus sinergias. En otras palabras, se podría afirmar que los beneficios públicos totales de la educación superior son mayores que la suma de los beneficios individuales (Willetts, 2015). A la luz de lo anterior, se puede afirmar que estas diferentes maneras de interpretar la educación superior como bien público están lejos de ser sencillas. Sin embargo, nuestra posición es que si bien es posible reconocer aspectos privados en la educación superior -los ingresos más altos por toda la vida son los más obvios-, sus propósitos deben verse más allá de las ganancias económicas que los graduados y la sociedad derivan de ella, ya que esto simplifica excesivamente los amplios compromisos y beneficios sociales que aportan las universidades al desarrollo cultural, económico y social.

\section{UN VÍNCULO SINGULAR ENTRE LA UNIVERSIDAD ESTATAL Y EL BIEN PÚBLICO}

El rol de la universidad estatal frente al bien público ha sido menos enfatizado en la literatura, debido a que culturalmente se da por sentado que estas instituciones en virtud de su naturaleza están orientadas hacia el. Aunque, Brunner y Peña (2011) indican que el vínculo entre la dimensión de lo público y las universidades estatales no necesariamente parece existir, puesto que independientemente de su carácter, todas las universidades producen en alguna medida bienes públicos y privados. Sostienen los autores que si bien existen razones poderosas para que el Estado financie la producción de bienes públicos, ese no es un motivo que actúe solo a favor de las instituciones estatales -es importante anotar que el análisis planteado se realiza en el contexto de la educación superior chilena, donde algunas universidades privadas reciben apoyo y financiamiento por parte del gobierno-. Más aún, Calhoun (2011) señala que la oferta creciente de las universidades privadas ha aportado al bien público por medio de un mayor acceso y, paradójicamente, la educación superior financiada con recursos públicos, en algunos casos, se ha convertido en un subsidio para las clases media y alta. En la misma línea, Collins (2017) añade que instituciones privadas pueden contribuir al bien público, haciendo que el conocimiento sea fácilmente accesible e incluso gratuito, mientras que las instituciones estatales pueden privatizar el conocimiento y retener el potencial público generado a partir de la actividad investigativa.

De otro lado, aludir desde lo público a una cierta esfera de la cultura o de la racionalidad humana, cuando este ámbito se constituye al margen y por oposición al Estado, permite afirmar que tampoco existe necesariamente una relación entre lo público y las universidades estatales (Brunner y Peña, 2011). Para Williams (2016) el conocimiento en forma de cultura ya no se presenta como una influencia civilizadora en la sociedad, ni desempeña un papel social que permita incluir a toda la sociedad en una visión cultural coherente. En contraste, Pusser et al. (2012) interpretan las universidades como esferas públicas, al señalar que estas instituciones deben ser sitios con la capacidad para sostener críticas independientes del Estado y muchas veces dirigidas hacia el, al tiempo que ofrecen opciones estratégicas. Sugieren que las universidades deben ser lugares de creación de conocimiento para la producción de bienes públicos esenciales para el bienestar social.

Concretamente, el vínculo privilegiado entre el bien público y lo estatal que se ha instalado fuertemente en la cultura, se puede encontrar a la sombra de la universidad moderna (Brunner y Peña, 2011). Y agregan los mismos autores que, en la visión que subyace de la universidad moderna, la universidad estatal adquiere un papel fundamental, en la medida en que ella es la única capaz de trascender la particularidad de grupos o intereses parciales y expresar, en cambio, la universidad del proyecto nacional que contribuye a: "homogeneizar la cultura y a configurar la nación; allí donde las clases y los grupos aspiran a reproducirse en una cadena simplemente endogámica y hereditaria, la universidad estatal aspira a hacer más diversas las élites; allí donde otras instituciones anhelan expresar puntos de vista particulares, las universidades estatales se dan a la tarea de crear un ámbito en el que todos esos puntos de vista pueden expresarse y debatir entre si". Por tanto, para Brunner y Peña trascender los puntos de vista puramente particulares, permitiría a las universidades estatales reclamar una atención preferencial por parte del Estado.

De este modo, el enorme potencial de la universidad estatal para contribuir al bien público lo encontramos en su promesa de justicia social, al brindar un mayor acceso a grupos de la población menos favorecidos o excluidos, y que históricamente han enfrentado grandes problemas de pobreza y desigualdad. Configurándose en una fuente de movilidad social que permite mejorar las condiciones de vida de personas independientemente de su origen social o capacidad financiera, aportando de esta forma a una sociedad más justa (Singh, 2014). Teixeira et al. (2017) manifiestan además que las políticas de acceso no sólo deben consistir en subsidios y préstamos estudiantiles, sino que también deben incluir regulaciones para prevenir la discriminación y mecanismos para elevar los niveles de aspiración de aquellos que tradicionalmente no 
consideran ingresar a la educación superior. En esta misma perspectiva, Nixon (2011) y más recientemente Williams, G (2016) afirma que la educación superior aporta al bien público si contribuye a promover una sociedad más igualitaria y se configura como un medio para lograr la justicia social a través de la movilidad individual. Sin duda, este aspecto adquiere una mayor relevancia para la universidad estatal, teniendo en cuenta que el sector de la educación en general presenta profundas desigualdades estructurales que se manifiestan en una estratificación institucional, reproducción de privilegios a través de mecanismos selectivos de admisión y la posterior consolidación de elites profesionales (Nixon, 2011). Esta estratificación institucional produce tanto ventajas como desventajas económicas que se refuerzan en el futuro, lo que conlleva a una reproducción intergeneracional de la desigualdad. En pocas palabras, las universidades desempeñan un papel ambiguo; aunque evidentemente proporcionan oportunidades que cambian la vida de las personas (Hall, 2012), también se convierten en un sistema que perpetúa desigualdades de acceso y de resultado, las cuales tienen un efecto muy perjudicial sobre las posibilidades de mejorar las condiciones de vida de una proporción significativa de jóvenes. Además, esas desigualdades se ven agravadas por la heterogeneidad de la calidad en la provisión del servicio (Nixon, 2015).

Conviene señalar que el papel de contribuir a la justicia social, no es el único que la universidad estatal tiene o podría jugar. De hecho, tradicionalmente se ha reconocido su compromiso y apoyo a comunidades locales y regionales, la preservación del conocimiento y su puesta a disposición de la comunidad, el avance del conocimiento a través de la investigación, el desarrollo de las artes y las humanidades, entre otros (Kezar, 2015). Pero también se ha enfatizado, en las últimas décadas, el rol en la creación, transmisión y difusión del conocimiento en un contexto de comercialización, a partir del cual se les asigna a las universidades la responsabilidad con el impulso, la ejecución y apoyo al desarrollo tecnológico de las empresas y de sus propios países (Schmal et al., 2010). Por lo que, en ese sentido, creemos que la justicia social representa una característica "específica" e inherente a la naturaleza de la universidad estatal, la cual debe ser reivindicada a la luz de que la distinción entre lo público y lo privado en la educación superior se ha desvanecido.

\section{LA UNIVERSIDAD ESTATAL FRENTE AL DESARROLLO DE LA CIENCIA COMO BIEN PÚBLICO}

Una nueva visión considera el bien público de la educación superior en términos de su contribución al aumento del conocimiento del individuo y de la sociedad en su conjunto (Brew, 2015). Previamente, Stiglitz (1999) había señalado que los bienes públicos más importantes producidos en la educación superior eran el conocimiento universal y la información: "Un teorema matemático conserva su valor sin importar cuántas veces o cuántas personas lo usen, podría servir como base para otras investigaciones e, incluso, estimular a otros a convertir una idea con valor comercial". En opinión de Dill (2015), la creación de nuevo conocimiento es a menudo costoso, sin embargo, una vez que ha sido creado, no impide que otras personas puedan beneficiarse de este. Por ejemplo, el software y ciertos tipos de diseños pueden ser replicados a un costo casi nulo (OCDE, 2013). En otras palabras y siguiendo a Marginson (2011), el conocimiento es la reivindicación única de la educación superior, debido a que es el núcleo de todo bien público y privado que se crea en el sector.

Ciertamente, la noción del conocimiento producido por las universidades como bien público admite algunas excepciones, ya que "ha sido un ingrediente de estatus y de elite y ha estado asociado múltiplemente con formas restrictivas y esotéricas" (Oyarzún, 2011). Dichas restricciones están asociadas con los lenguajes especializados, las condiciones de acreditación como miembro de una comunidad de conocimiento, los costos relacionados con la disponibilidad de los saberes bajo cualquiera de formas que ella pueda asumir, los derechos de propiedad intelectual y las patentes, que proporcionan medios para excluir a otros de los resultados de la investigación, al menos durante un cierto periodo de tiempo. Como resultado de ello, la tradición de la cultura y de la educación como bienes públicos ha comenzado a privatizarse con el ascenso de las llamadas universidades de investigación (Morandé, 2011), denominadas por Salmi (2009) universidades de rango mundial, que son reconocidas por la superioridad de sus resultados: graduados excepcionalmente calificados y con una alta demanda en el mercado laboral, investigaciones de vanguardia con publicaciones en las principales revistas científicas y, en el caso de instituciones orientadas hacia la ciencia y la tecnología, contribuyen con innovaciones tecnológicas. Estas universidades según Morandé "tienen la capacidad de transformar el saber en información y hacerlo intransparente para asociarle la expectativa de un valor comercial agregado".

En la línea de ideas anterior, Moriarty (2011) sostiene que la ciencia como un bien público está siendo socavada. Señala este autor que las normas propuestas por Robert K. Merton en 1942, son todavía un conjunto de principios rectores que pueden utilizarse para distinguir ampliamente la ciencia académica tradicional de la I + D comercializada, emprendedora y cercana al mercado, la cual se promueve cada vez más en el entorno de la educación superior. Específicamente, las normas de la ciencia incluyen los conceptos de: a) el universalismo: las afirmaciones de la verdad científica son independientes de las cualidades personales, nacionales o de los orígenes de sus descubridores; b) el comunalismo: mediante el cual los 
resultados de la ciencia son productos de la colaboración social y se asignan a la comunidad. Estos dos conceptos forman el fundamento de la ciencia abierta, que requiere de la publicación y difusión accesible de los descubrimientos científicos y sus aplicaciones (Triggle y Triggle, 2017). David (2003), quien según la OCDE (2015) acuñó el término "ciencia abierta", agregó que la divulgación completa de los hallazgos y los métodos, constituyen un aspecto clave del programa cooperativo y comunitario de la investigación, debido a que contribuye a legitimar y aumentar la rapidez en la validación de los hallazgos, reduciendo al mismo tiempo la duplicación de los esfuerzos en esta actividad.

Es importante mencionar que organizaciones intergubernamentales como la OCDE, la UNESCO, la UE y el Banco Mundial en los últimos años han estado desarrollando estrategias y políticas relacionadas con la promoción de la ciencia abierta, la cual incluye la interoperabilidad de la infraestructura científica, las metodologías abiertas y compartidas de investigación (como las aplicaciones abiertas y el código informático). De acuerdo con la OCDE (2015), la ciencia abierta se refiere a los esfuerzos de investigadores, gobiernos, organismos de financiación de la investigación o la propia comunidad científica para hacer que la producción de investigación financiada con fondos públicos - publicaciones y datos de investigación - sea más ampliamente accesible en formato digital a la comunidad científica, al sector empresarial o a la sociedad en general. En síntesis, la ciencia abierta en la era de la información sostiene la idea de que el conocimiento creado a partir de la investigación financiada con fondos públicos tiene características de un bien público. Esto significa que dicho conocimiento debe ser ampliamente accesible para el uso de cualquier persona y sin costo adicional una vez que se haga público, generando de este modo retornos sociales más amplios. Por tanto, si las universidades estatales están realmente interesadas en fortalecer su papel público, no pueden ignorar estas demandas de la sociedad y, en razón de su naturaleza, deben tener un papel activo en la implementación de políticas y prácticas que promuevan de manera libre el acceso al conocimiento generado de sus actividades de investigación.

\section{ALGUNOS RETOS Y DIMENSIONES DEL BIEN PÚBLICO EN LA EDUCACIÓN SUPERIOR COLOMBIANA}

El Sistema de Educación Superior Colombiano en los últimos años ha presentado cambios importantes. En primer lugar, el aumento de cobertura es una muestra de la expansión del sector y de su importancia para el desarrollo económico, social y cultural del país (aumentó del 30\%, en el 2006, al 51.2\%, en 2016, de acuerdo con el Ministerio de Educación Nacional). No obstante, según cifras del 2015, existen grandes brechas de acceso, pues solo el $57 \%$ del total de municipios del territorio nacional poseían al menos un estudiante matriculado en programas de educación superior. De este modo, la mayor parte de la oferta se encuentra fuertemente concentrada en algunos departamentos o ciudades del país. Por ejemplo, entre Bogotá, Antioquia, Valle del Cauca, Atlántico y Santander, están el 64.6\% de los matriculados frente al 35.4\% restante en los demás departamentos.

En segundo lugar, como un indicador de la calidad de instituciones y programas, es conveniente subrayar que, en 2016, 47 Instituciones de Educación Superior -IES- se encontraban acreditadas (de un total de 187), de las cuales 32 eran privadas y 15 estatales. En cuanto a los programas, se pasó de 895 programas acreditados en 2015 a 1.063 en 2016, lo que representa un incremento del 19\% (el total de programas en los diferentes niveles de formación fue de 11.213). Lo anterior, permite inferir que la acreditación cubre un bajo porcentaje de instituciones y programas, lo cual implica la necesidad de realizar mayores esfuerzos en este sentido.

En tercer lugar, en 2015, el 31\% de los profesores poseían título de pregrado, el 30\% de especialización, con título de maestría era del $29 \%$, y sólo un $6 \%$ tenía título de doctorado. Cabe anotar, sin embargo, que el número de profesores con formación doctoral en el 2015 es superior (9.477) al que había en el 2010 (4.526). Para el mismo año, 2015, los catedráticos representaban el $48 \%$, los de tiempo completo un $30 \%$ y los vinculados por medio tiempo un $22 \%$. Si bien los profesores de tiempo completo han aumentado, el mayor crecimiento en el número de profesores se presenta en los catedráticos, constituyendo la categoría más grande de profesores en el país (71.937). Hecho que afecta la potencialidad de las IES para el desarrollo de capacidades de investigación y en los procesos de calidad.

En cuarto lugar, se evidencia un mejor desarrollo de las capacidades científicas y tecnológicas, pues en período 2006 el número de estudiantes graduados en programas de doctorado fue de 91 y 3.286 de maestría, mientras que para 2015, se otorgaron respectivamente 466 y 14.602 títulos. Finalmente, se puede resaltar un creciente interés en la dimensión de la internacionalización por parte de las IES y de las políticas públicas nacionales. Por su puesto, estos son solo algunos de los avances y retos que enfrenta la educación superior colombiana, pero que consideramos son representativos de la evolución positiva que ha tenido el sector en la última década. 
En Colombia la educación superior como bien público ha sido poco interrogada. No obstante, en su marco jurídico es posible identificar algunos aspectos que podrían interpretarse de un modo amplio como bienes públicos. A saber, la Ley 30 de 1992 (Ley de educación superior en Colombia) pone de manifiesto la orientación hacia el logro de los siguientes objetivos (art. 6): la formación integral de los colombianos, la creación, el desarrollo y la transmisión del conocimiento, promover su utilización en todos los campos para solucionar las necesidades del país, prestar a la comunidad un servicio con calidad, ser factor de desarrollo científico, cultural, económico, político y ético a nivel nacional y regional, entre otros. Asimismo, la Constitución Política expresa que a través de la educación se busca garantizar "el acceso al conocimiento, a la ciencia, a la técnica y a los demás valores de la cultura" (art. 67). Desde este enfoque jurídico, la educación superior es considerada como un derecho de la persona y un servicio público con función social. También estipula que: "la educación formará al colombiano en el respeto a los derechos humanos, a la paz y a la democracia y en la práctica del trabajo y la recreación, para el mejoramiento cultural, científico, tecnológico de la nación y para la protección del ambiente". De este modo, la Constitución le asigna una función social a la educación superior colombiana en sus propósitos de acceso a la cultura, a la ciencia libre y la tecnología, incluyendo además la autonomía universitaria. Aún más, como un derecho presupone la eliminación de barreras que obstaculicen su goce efectivo (Arango, 2014).

Igualmente, en los últimos años, a raíz del fallido intento en 2011 por reformar la Ley 30, se han generado reflexiones en torno a las bases y principios que deben orientar una nueva política pública de educación superior. El Consejo Nacional de Educación Superior (CESU, 2014), en un trabajo denominado "Acuerdo por lo Superior 2034: Propuesta de Política Pública para la excelencia de educación superior en Colombia en el escenario de la paz", señala que "la educación superior incluyente y de calidad constituye la verdadera garantía para convivir en una Colombia que pueda superar el conflicto y reconstruirse positivamente". A este respecto, es importante mencionar el compromiso que han asumido algunas universidades en el marco del postconflicto (por ejemplo, Red de Universidades por la Paz), mediante la asignación de becas y programas de formación a excombatientes y víctimas, en áreas rurales fuertemente golpeadas por conflicto armado y donde las tasas de acceso a la educación han sido las más bajas del país. El objetivo de estas estrategias y programas es el de ayudar a fomentar la convivencia y la integración de los distintos actores de la guerra. En esta perspectiva, sería valioso profundizar en el concepto de "justicia curricular", propuesto por Zembylas (2017), un enfoque estratégico basado en los intereses de los grupos de estudiantes menos favorecidos.

Por su parte, la Asociación Colombiana de Universidades (ASCUN, 2012) propone el "desarrollo humano sostenible y la transformación de la sociedad" como eje de una política pública de la educación superior; lo cual implica concebir dicho desarrollo como un proceso de cambio en las relaciones entre los sistemas, instituciones y procesos que tienen que ver con lo social, lo valorativo, lo productivo y el uso y explotación de los recursos naturales. En relación con la transformación de la sociedad, señala la importancia de "construir capital social, que conlleve a la creación de instituciones sociales capaces de generar bienes públicos y confianza, en el marco de la equidad, el respeto por los derechos humanos, la seguridad ciudadana y la paz". En esta misma línea, el CESU (2014) afirma que dicha educación debe ser inclusiva, participativa, pertinente, integrada con otros sectores sociales, debidamente sostenible, consciente de su responsabilidad directa con lo social y con estándares de calidad en todo el territorio nacional. Lo anterior, sugiere la necesidad de incorporar nuevas dimensiones orientadoras en la formulación de una nueva política pública de educación superior en Colombia, en la que el creciente marco teórico del bien público constituye un punto de referencia valioso por explorar a fin de revitalizar la misión pública de las universidades.

De otro lado, en relación a la concepción de la investigación en Colombia, inquieta el hecho de que la ley de ciencia y tecnología (Ley 1286 de 2009) haga énfasis en que la innovación y generación de conocimiento deben fortalecer la competitividad y el desarrollo económico del país (art. 1): "El objetivo general de la presente ley es fortalecer el Sistema Nacional de Ciencia y Tecnología y a Colciencias para lograr un modelo productivo sustentado en la ciencia, la tecnología y la innovación, para darle valor agregado a los productos y servicios de nuestra economía y propiciar el desarrollo productivo y una nueva industria nacional". En otras palabras, la norma supedita los múltiples propósitos de la ciencia a los intereses económicos de las empresas, bajo el paradigma neoliberal, sin darle el valor real a la generación del conocimiento en sí mismo. No se trata de reprobar las relaciones de las universidades -que realizan la mayor parte de actividades de I+D en el paíscon el sector empresarial, pues es claro que la torre de marfil ha desaparecido, de hecho, el mundo postindustrial del siglo XXI las sitúa como centros de conocimiento, investigación e innovación que están a la vanguardia de la globalización (Altbach et al., 2016). Por ello, quizás lo que se debería contemplar son formas que abarquen pero al mismo tiempo trasciendan los imperativos económicos. En igual sentido, Singh (2012) afirma que una mejor comprensión de las diferentes implicaciones del "mercado" en la educación superior permitiría distinguir qué dimensiones son inevitables, qué se debe disputar o mediar y qué debe ser apoyado en un entorno de transformación. Finalmente, es importante reconocer el alto nivel de generalidad e, incluso, de confusión en los valores y propósitos asociados con el bien público, los cuales podrían ser vistos sólo como una declaración retórica. Sin embargo, creemos que no deberían subestimarse, pues representan un punto de referencia valioso de mejores aspiraciones para la educación superior. 


\section{CONCLUSIONES}

De acuerdo con el análisis expuesto, se pueden deducir las siguientes conclusiones:

i) una de las principales funciones de la universidad estatal, inherente a su naturaleza, en su papel de servir al bien público se encuentra en su promesa de justicia social, al brindar acceso a grupos de la población menos favorecidos o excluidos, aportando de esta manera a una sociedad más justa; ii) el conocimiento generado a partir de la investigación financiada con fondos gubernamentales tiene las características de un bien público, lo cual implica que la universidad estatal debe promover el libre el acceso a dicho conocimiento para todas las partes interesadas, ello constituye el fundamento de la ciencia abierta; iii) dentro de los principales desafíos de la educación superior colombiana se encuentran: continuar aumentando el acceso con equidad y calidad en todas las regiones del país con el fin de reducir las actuales brechas existentes, aumentar la vinculación de docentes de tiempo completo, promocionar la formación avanzada y mejorar el desarrollo de las capacidades científicas y tecnológicas.

\section{REFERENCIAS}

Altbach, P.G., Reisberg, L. y Rumbley, L.E., Tracking a Global Academic Revolution, in Global perspectives on higher education by Altbach, P.G., pp. 25-37, Johns Hopkins University Press, Baltimore, USA (2016)

Arango, R., La educación superior en el orden democrático constitucional, en La educación superior: retos y perspectivas por Orozco, L.E., pp. 201-250, Ediciones Uniandes, Bogotá, D.C., Colombia (2014)

ASCUN. Desarrollo humano sostenible y transformación de la sociedad. Política pública para la educación superior y agenda de la Universidad, de cara al país que queremos (en la web: https://goo.gl/1f6uNT, acceso: 25 de mayo 2016), Asociación Colombiana de Universidades, Bogotá D.C., Colombia (2012)

Baum, S., y McPherson, M., Is education a public or a private good? (en la web: http://chronicle.com/blogs/innovations/iseducation-a-public-good-or-a-private-good/28329, acceso: 17 de septiembre de 2017), The Chronicle of Higher Education, Washington, D.C., USA (2011)

Brew, A., The paradoxical University and the Public Good, in Higher education as a public good: Critical perspectives on theory, policy and practice by Filippakou, O. y Williams, G., pp. 97-112, Peter Lang Publishing, New York, USA (2015)

Brunner, J.J. y Peña, C., La dialéctica público/privado entre el medioevo y la globalización, en El conflicto de las universidades: entre lo público y lo privado por Brunner, J.J. y Peña, C., pp. 9-61, Ediciones Universidad Diego Portales, Santiago, Chile (2011)

Brunner, J.J., Lo Público de la Universidad a la Luz de la Historia Larga, https://goo.gl/S6vPa3, ISSN: 0718-1361, Revista Interdisciplinaria de Filosofía y Psicología 11(37), 49-55 (2016)

Calhoun, C., The Public Mission of the Research University, in Knowledge matters: The public mission of the research university by Rhoten, D. y Calhoun, C., pp. 1-33, Columbia University Press, New York, USA (2011)

CESU. Acuerdo por lo Superior 2034: Propuesta de política pública para la excelencia de la educación superior en Colombia en el escenario de la paz (en la web: https://goo.gl/f63P2x, acceso: 25 de junio de 2016), Ministerio de Educación Nacional de Colombia, Bogotá, D.C. (2014)

Collins, C.S., Academic Public Good, In University-Community Engagement in the Asia Pacific by Collins C.S., pp. 1-11, Palgrave Macmillan, California, USA (2017)

David, P., The Economic Logic of "Open Science" and the Balance between Private Property Rights and the Public, Domain in Scientific Data and Information (en la web: http://www.nap.edu/catalog/10785.html, acceso: 07 de Agosto 2017), The National Academies Press, Washington, DC (2003)

Dill, D.D., Assuring the Public Good in Higher Education: Essential Framework Conditions and Academic Values, in Higher education as a public good: Critical perspectives on theory, policy and practice by Filippakou, O. y Williams, G., pp. 141162, Peter Lang Publishing, New York, USA (2015)

Enders, J. y Jongbloed, B., Public-Private Dynamics in Higher Education: Expectations, Developments and Outcomes (en la web: https://goo.gl/YqV9cp, acceso: 07 de febrero 2017), Transcript Verlag, Bielefeld, Germany (2008)

Hall, M., Public good and private benefits of higher education, in Higher education for the public good: Views from the south by Leibowitz, B., pp 17-29, Trentham Books, Stoke on Trent, England (2012)

Hensley, B., Galilee-Belfer, M. y Lee, J. J., What is the greater good? The discourse on public and private roles of higher education in the new economy, doi: 10.1080/1360080X.2013.825416, Journal of Higher Education Policy and Management, 35(5), 553-567 (2013)

Kaul, I., Grunberg, I. y Stern, M., Global Public Goods: International Cooperation in the 21st Century by Kaul, I.; Grunberg, I. y Stern, M., pp. 1-20, Oxford University Press, New York, USA (1999)

Kezar, A., Challenges for Higher Education in serving the Public Good, in Higher education for the public good: Emerging voices from a national movement by Kezar, A., Chambers, A. y Burkhardt, J. C., pp. 56-73, John Wiley \& Sons, San Francisco, USA (2015) 
Longden, B. y Bélanger, C., Universities: Public good or private profit, doi: 10.1080/1360080X.2013.825417, Journal of Higher Education Policy and Management, 35(5), 501-522 (2013)

Marginson, S., The Public/Private Divide in Higher Education: A Global Revision, doi:10.1007/s10734-005-8230-y, Higher Education, 53(3), 307-333 (2007)

Marginson, S., Higher Education in the Global Knowledge Economy, doi: 10.1016/j.sbspro.2010.05.049, Procedia- Social and Behavioral Sciences, 2(5), 6962-6980 (2010)

Marginson, S., Higher Education and Public Good, doi: 10.1111/j.1468-2273.2011.00496.x, Higher Education Quarterly, 65(4), 411-433 (2011)

Marginson, S., The "Public" Contribution of Universities in an Increasingly Global World, in Universities and the Public Sphere: Knowledge Creation and State Building in the Era of Globalization by Pusser, B., Kempner, K. y otros dos autores, pp. 22-33, Routledge, New York, USA (2012)

McMahon, W.M., Higher learning, greater good: The private and social benefits of higher education, 119-180, The John Hopkins University Press, Baltimore, USA (2009)

Morandé, P., Lo público y lo privado en el sistema universitario chileno: visión desde la cultura, en El conflicto de las universidades: entre lo público y lo privado por Brunner, J.J. y Peña, C., pp. 65-82, Ediciones Universidad Diego Portales, Santiago, Chile (2011)

Moriarty, P., Science as a Public Good, in A manifesto for the public university by Holmwood, J., pp. 56-73, Bloombury Academic, London, UK (2011)

Nixon, J., Higher education and the public good: Imagining the University, 1-17, Bloomsbury, London, Great Britain (2011)

Nixon, J., Inequality and the erosion of the public good, in Higher education as a public good: Critical perspectives on theory, policy and practice by Filippakou, O. y Williams, G., pp. 163-177, Peter Lang Publishing, New York, USA (2015)

OCDE. "New sources of growth: knowledge-based capital", Key Analysis and Policy Conclusions. Synthesis (en la web: https://goo.gl/4WY8SC, acceso: 12 de julio 2017), OECD Publishing (2013)

OCDE. "Making Open Science a Reality", (en la web: http://dx.doi.org/10.1787/5jrs2f963zs1-en, acceso: 31 de julio de 2017), OECD Publishing, Paris (2015)

Oyarzún, P., Las humanidades lo público y la Universidad, en El conflicto de las universidades: entre lo público y lo privado por Brunner, J.J. y Peña, C., pp 111-131, Ediciones Universidad Diego Portales, Santiago, Chile (2011)

Popp, E. y Paradise, C., Introduction, in The University Under Pressure by Popp, E. y Paradeise, C., pp. 1-22, Emerald Group Publishing Limited, Bingley, UK (2016)

Pusser, B., Power and Authority in the Creation of a Public Sphere Through Higher Education, in Universities and the Public Sphere: Knowledge Creation and State Building in the Era of Globalization by Pusser, B., Kempner, K. y otros dos autores, pp 34-41, Routledge, New York, USA (2012)

Salmi, J., El desafío de crear universidades de rango mundial, (en la web: https://goo.gl/gE5ST1, acceso: 07 de febrero 2017), Banco Mundial, Washington, D.C. (2009)

Santiago, P., Tremblay, K. y otros dos autores, Tertiary education for the knowledge society (en la web: https://goo.gl/fVHZ9m, acceso 01 de junio 2017), OECD, Paris (2008)

Schmal, R., López, S., Cabrales, F. y Acuña, D., Modelado de Procesos de Negocio para la Gestión de Patentes en Universidades, doi: https://dx.doi.org/10.4067/S0718-07642010000600013, Información Tecnológica, 21(6), 113-124 (2010)

Schoenenberger, A.M., Are higher education and academic research a public good or a public responsibility? A review of the economic literature, in The Public Responsibility for Higher Education and Research by Weber, L. y Bergan, S., pp. 45-94, Council of Europe Publishing, Strasbourg, Francia (2005)

Scott, P., Higher Education, the Public Good and the Public Interest, in Higher education as a public good: Critical perspectives on theory, policy and practice by Filippakou, O. y Williams, G., pp. 41-58, Peter Lang Publishing, New York, USA (2015)

Singh, M., Re-inserting the 'public good' into higher education transformation, in Higher education for the public good: Views from the south by Leibowitz, B., pp. 1-16, Trentham Books, Stoke on Trent, England (2012)

Singh, M., Higher education and the public good: precarious potential? http://www.ufs.ac.za/ActaAcademica; ISSN: $0587-$ 2405, Rev. Acta Academica, 46(1), 98-118 (2014)

Sobrinho, J. D., Conferencia Mundial sobre la Educación Superior-2009: La nueva dinámica de la educación superior y la investigación para el cambio social y el desarrollo (en la web: https://goo.gl/RrrYQj, acceso: 07 de junio 2017), UNESCO, Sede de la UNESCO, París, 5-8 de julio (2009)

Stiglitz, J.E., Knowledge as a global public good, in Global Public Goods: International Cooperation in the 21st Century by Kaul, I., Grunberg, I. y Stern, M., pp. 308-325, Oxford University Press, New York, USA (1999) 
Teixeira, P., S. Kim y otros dos autores, Introduction, in Rethinking the Public-Private Mix in Higher Education: Global Trends and National Policy Challenges by Teixeira, P., Kim, S. y otros dos autores, pp. xi-xiv, Sense Publishers, Rotterdam, The Netherlands (2017)

Triggle, C. R., y Triggle, D.J., From Gutenberg to Open Science: An Unfulfilled Odyssey, doi:10.1002/ddr.21369, Drug Development Research, 78(1), 3-23 (2017)

Willetts, D., Issues and Ideas on Higher Education: Who benefits? Who pays? (en la web: https://goo.gl/MHBYWR, acceso 09 de Agosto 2017), King's College London, London, UK (2015)

Williams, J., A critical exploration of changing definitions of public good in relation to higher education, doi: 10.1080/03075079.2014.942270, Studies in Higher Education, 41(4), 619-630 (2016)

Williams, G., Higher education: Public good or private commodity? doi:10.18546/LRE.14.1.12, London Review of Education, 14(1), 131-142 (2016)

Zembylas, M., Higher education for the public good in post-conflict societies - curricular justice and pedagogical demands: a response from Cyprus and South Africa, doi: 10.1080/07294360.2017.1249071, Higher Education Research \& Development, 36(1), 36-42 (2017) 